\title{
Pinning Stabilization of Complex Networks Coupled with Time Delay and Disturbed with Stochastic Noise
}

\author{
Chun-Xia Fan, Yu Gu, and Qingyang Wei \\ College of Automation, Nanjing University of Posts and Telecommunications, Nanjing 210003, China \\ Correspondence should be addressed to Chun-Xia Fan; njfcx@126.com
}

Received 21 August 2013; Revised 25 December 2013; Accepted 11 March 2014; Published 6 April 2014

Academic Editor: James Lam

Copyright (c) 2014 Chun-Xia Fan et al. This is an open access article distributed under the Creative Commons Attribution License, which permits unrestricted use, distribution, and reproduction in any medium, provided the original work is properly cited.

\begin{abstract}
A pinning stabilization problem of complex networks with time-delay coupling is studied under stochastic noisy circumstances in this paper. Only one controller is used to stabilize the network to the equilibrium point when the network is connected and the minimal number of controllers is used when the network is unconnected, where the structure of complex network is fully used. Some criteria are achieved to control the complex network under stochastic noise in the form of linear matrix inequalities. Several examples are given to show the validity of the proposed control criteria.
\end{abstract}

\section{Introduction}

Complex networks have been a major research topic and attracted increasing attention from various fields including physics, biology, sociology, and engineering. Many real phenomena can be described as complex networks, such as the World Web, telephone call graphs, and social organization. Recently, the stabilization and synchronization problem and stabilization problem of complex network have become more and more important.

In fact, the synchronization problem is a special stabilization one since it can be converted into the stabilization problem of the error system between the complex network and the synchronization manifold [1-3]. Specially, the synchronization problem is a stabilization one when the synchronization manifold is an equilibrium orbit. So in this paper, we study the stabilization problem of complex networks which can be extended to the synchronization problem. Many contributions on complex network synchronization or stabilization are derived on the basis of the inner coupling strength adjustable [4-6]; that is, the whole network can synchronize or stabilize by itself.

However, it is true that the inner coupling strength sometimes cannot be adjustable for a complex network. Consequently, the whole network cannot be synchronized or stabilized by itself [7]. Therefore, some additional controllers have to be applied to force the network to be synchronized or stabilized. How many controllers are added to stabilize the complex network? Adding the controllers to all the nodes is the most simple but costly and impossible due to the complexity of network. To reduce the number of controlled nodes, some local feedback injections are applied to a fraction of networks nodes, which is called pinning control [8-12]. Wang and Chen found that specific pinning of the nodes with larger degree required a smaller number of controlled nodes than the random pinning for a scale-free network [8]. Li et al. proposed the virtual control method for microscopic dynamics throughout the process pinning control to stabilize a complex network to its equilibriums [13]. Topology is important for the network synchronization. Chen et al. used a single controller to synchronize the complex network with irreducible topology matrix and a minimal number of controllers for network with reducible topology matrix [9]. Lu et al. extended the results obtained in [9] to linearly coupled neural network perturbed by stochastic noise and pinning stabilized the neural networks to homogenous solutions [14]. In $[9,14]$, the inner coupled matrix of network must be diagonal and positive. How to deal with the more general coupled way? Zhou et al. proposed a scheme of determining the number of pinning controlled nodes for general complex networks with positive definite inner coupling [15]. 
All of the above-mentioned contributions focus on the complex networks coupled with no time delaying. However, there often exists time delaying when the signals are transmitted over networks $[10,11,16-20]$. Therefore, we have to consider the complex network coupled with time delaying. For the coupling matrix being irreducible, the pinning stabilization criteria were proposed in the form of liner matrix inequality for a complex network coupling time delaying $[10,11]$. However, it is regretted that the method of selecting the controlled node number is not given. All of the nodes needed to be controlled in a complex network coupled with time delaying $[16,17,20]$. How to select the controlled node for a complex network coupled with time delaying?

Motivated by the above discussion, we study the pinning stabilization problem on complex networks coupled with time delaying and disturbed by the stochastic noise from three kinds of topology matrices: symmetrical and irreducible, asymmetrical and irreducible, and $m$ reducible. Only one controller is used to stabilize a complex network coupled with irreducible matrix and only $m$ controllers are used to a complex network coupled with $m$-irreducible matrix. Some stabilization criteria are derived by using Llyapunov stability theorem and stochastic analysis.

The rest of this paper will be organized as follows. In Section 2, the model formulation of linearly delaycoupled complex networks with a noise perturbation and some preliminaries will be presented. In Section 3, the pinning stabilization problem of complex networks with, respectively, symmetric irreducible coupling matrix, asymmetric irreducible coupling matrix, and $m$-reducible coupling matrix will be studied. Some criteria will be derived in terms of linear matrix inequalities (LMIs) to guarantee the success of designed controllers in Section 3. In Section 4, several computer numerical simulations will be given to show the effectiveness of the proposed stabilization approach, and Section 5 concludes the investigation.

Notations. The standard notations will be used in this paper. Throughout this paper, for real symmetric matrices $X$ and $Y$, the notation $X \leq Y(X<Y)$ means that the matrix $X-Y$ is negative semidefinite (negative definite). $I_{n}$ is the identity matrix of order $n$. We use $\lambda_{\min }(\cdot)$ and $\lambda_{\text {max }}(\cdot)$ to respectively denote the minimum and maximum Eigen value of a real symmetric matrix. The notation $\|X\|$ denotes Euclidean norm of vector $X$, and $\max _{i}\left\{x_{i}\right\}$ means the maximum element of vector $X$. $\mathfrak{R}$ denotes the set of real numbers. $\mathfrak{R}^{n \times n}$ denotes the $n \times n$ real matrices. $\operatorname{diag}\{\cdots\}$ stands for a blockdiagonal matrix. The superscript " $T$ " represents the transpose of the matrix. $E\{\cdot\}$ denotes the mathematical expectation operator.

\section{Model Formulation and Some Preliminaries}

Consider a general complex dynamical network consisting of $N$ identical nodes with linearly diffusive time-delayed couplings and perturbed with stochastic noise, which is described as follows:

$$
\begin{aligned}
d x_{i}(t)= & {\left[f\left(x_{i}(t)\right)+\sum_{j=1}^{N} c_{i j} A x_{j}(t-\tau)+u_{i}(t)\right] d t } \\
& +g\left(t, x_{i}(t), x_{i}(t-\tau)\right) d \omega_{i}(t) \quad i=1,2, \ldots N
\end{aligned}
$$

where $x_{i}(t)=\left(x_{i 1}(t), x_{i 2}(t), \ldots, x_{i n}(t)\right)^{T} \in \mathfrak{R}^{n}$ is the state vector of $i$ th node, $f$ is a nonlinear vector function, $A=$ $\operatorname{diag}\left\{a_{1}, a_{2}, \ldots, a_{n}\right\} \in R^{n \times n}$ is the inner connecting weight matrix between two connected nodes, $C=\left(c_{i j}\right)_{N \times N}$ is the coupling configuration matrix of the drive complex network, and the elements $c_{i j}$ in matrix $C$ are defined as follows: if there is a connection from node $j$ to node $i(i \neq j)$, then $c_{i j}>0$, otherwise $c_{i j}=0(i \neq j)$, and the diagonal elements of $C$ are satisfied as follows: $c_{i i}=-\sum_{j=1, j \neq i}^{N} c_{i j} i=1,2, \ldots, N$. $\tau$ denotes the time delay of the networks coupling, $W(t)=$ $\left(\omega_{1}(t), \omega_{2}(t), \ldots, \omega_{m}(t)\right)^{T} \in \mathfrak{R}^{m}$ is an $m$-dimensional Brownian motion defined on a complete probability space $(\Omega, F, P)$ with filtration $\left\{F_{t}\right\}_{t \geq 0}$ satisfying the usual conditions (i.e., the filtration contains all $P$-null sets and is right continuous). Here, the white noise $d \omega_{i}(t)$ is independent of $d \omega_{j}(t)$ for $i \neq j$, and $g: \mathfrak{R}^{+} \times \mathfrak{R}^{n} \times \mathfrak{R}^{n} \rightarrow \mathfrak{R}^{n \times m}$ is named the noise intensity function matrix. The stabilization controllers $u_{i}(t)(i=1,2, \ldots, l, l \leq N)$ will be determined and the number of the controlled nodes will be given in Section 3, which can stabilize the network (1) to equilibrium in mean square.

For the function $f(\cdot)$, one has the following assumption.

Remark 1. Network (1) is different from the one considered in [9-11]. Here, network (1) is disturbed by a stochastic noise and coupled with time delaying. However, the transmitting time delaying is not considered in [9] and the stochastic perturbation is not considered in $[10,11]$.

Assumption 2. The function $f(\cdot)$ of the complex networks satisfies the following Lipschitz condition:

$$
\begin{gathered}
\left\|f_{i}(x)-f_{i}(y)\right\| \leq Q_{i}\|x-y\|, \\
\forall x, y \in \Re^{n}, \quad i=1,2, \ldots, N,
\end{gathered}
$$

where $Q_{i}$ is a positive constant for $i=1,2, \ldots, n$. For convenience, let $Q=\operatorname{diag}\left\{Q_{1}, Q_{2}, \ldots, Q_{n}\right\}$.

Assumption 3. The noise intensity function matrix $g$ is uniformly Lipschitz continuous satisfying the following linear growth condition:

$$
\begin{aligned}
& \operatorname{trace}\left[g^{T}\left(t, x_{i}(t), x_{i}(t-\tau)\right) \cdot g\left(t, x_{i}(t), x_{i}(t-\tau)\right)\right] \\
& \quad \leq\left\|M_{1} x_{i}(t)\right\|^{2}+\left\|M_{2} x_{i}(t-\tau)\right\|^{2},
\end{aligned}
$$

where $M_{1}$ and $M_{2}$ are known constant matrices with compatible dimensions. 
Remark 4. Condition (3) on the noise density function matrix guarantees that the elements of $g\left(t, x_{i}(t), x_{i}(t-\tau)\right)$ are bounded and differential [21]. This assumption has been widely used for the stability analysis of stochastic differential equations $[22,23]$.

Assumption 5. The inner coupling matrix $A$ is diagonal and positive definite; that is, $A=\operatorname{diag}\left\{a_{1}, a_{2}, \ldots, a_{n}\right\}>0$.

Remark 6. Assumption 5 has been used in neural networks and complex networks $[9,14,20]$. This diagonal condition is easy and convenient to achieve the controller. And this diagonal condition is extended to the general coupling since a lot of matrix can be diagonalized.

The following definitions and lemmas are required for the derivation of our main results in this paper.

Definition 7 (see [14]). Matrix $C$ is said to be reducible if it can be transformed to a matrix of the form $\left[\begin{array}{cc}C_{1} & C_{3} \\ O & C_{2}\end{array}\right]$ by the same permutation of the rows and columns, where $C_{1}$ and $C_{2}$ are square matrices and $O$ is a null matrix.

Definition 8 (see [14]). The pinning controlled network (1) is said to be globally exponentially stabilized at the original point in mean square, if for any given initial condition, there exists positive constant $M_{0}$ and $\eta$ such that

$$
\begin{array}{r}
E\left\{\left\|x_{i}(t)\right\|^{2}\right\} \leq M_{0} e^{-\eta t} E\left\{\sup _{s \in[-\tau, 0]} \sum_{i=1}^{N}\left\|x_{i}(s)\right\|^{2}\right\} \\
(i=1,2, \ldots, N),
\end{array}
$$

where $E\{\cdot\}$ is the mathematical expectation.

Definition 9 (see [24]). For an $N \times N$ irreducible square matrix $H$ with nonnegative off-diagonal elements, $\beta(H)$ is defined as follows: $H$ is decomposed uniquely as $H=L+F$, where $L$ is a zero row sum matrix and $F$ is a diagonal matrix. Let $\varphi=\left(\varphi_{1}, \varphi_{2}, \ldots, \varphi_{N}\right)^{T}$ be the unique normalized left eigenvector of $L$ with respect to the Eigen value zero satisfying $\max _{k}\left\{\varphi_{k}\right\}=1$, and $\Phi=\operatorname{diag}\{\varphi\}$. Then we can obtain $\beta(H)=$ $\lambda_{\max }\left(\Phi H+H^{T} \Phi\right)$.

Definition 10 (see [24]). Consider a reducible matrix $C$ of order $N$. The matrix is $N$-reducible if it is diagonal. For $1 \leq$ $m<N$, the matrix $C$ is $m$-reducible if it is not $(m+1)$ reducible and it can be rewritten in the following Frobenius normal form after certain permutations:

$$
C=\left[\begin{array}{cccccccc}
C_{1} & C_{12} & \cdots & C_{1 k} & C_{1, k+1} & C_{1, k+2} & \cdots & C_{1, k+m} \\
0 & C_{2} & \cdots & C_{2 k} & C_{2, k+1} & C_{2, k+2} & \cdots & C_{2, k+m} \\
\vdots & \vdots & \ddots & \vdots & \vdots & \vdots & \vdots & \vdots \\
0 & 0 & \cdots & C_{k} & C_{k, k+1} & C_{k, k+2} & \cdots & C_{k, k+m} \\
0 & 0 & \cdots & 0 & C_{k+1} & 0 & \cdots & 0 \\
0 & 0 & \cdots & 0 & 0 & C_{k+2} & \cdots & 0 \\
\vdots & \vdots & \vdots & \vdots & \vdots & \vdots & \ddots & \vdots \\
0 & 0 & \cdots & 0 & 0 & 0 & \cdots & C_{k+m}
\end{array}\right]
$$

where $C_{r}(r=1,2, \ldots, k+m)$ are square irreducible matrices, and for each $r \leq k$, there exists $s>r$ such that $C_{r s} \neq 0$.

Lemma 11 (see [25]). Let $C=\left(c_{i j}\right)_{n \times n}$ be an $n \times n$ matrix with elements such that $\left|c_{i i}\right| \geq \sum_{j=1, j \neq i}^{n}\left|c_{i j}\right|(i=1,2, \ldots, n)$ with equality in at most $n-1$ cases. Assume that the matrix $C$ is nonsingular, that is to say, $|C| \neq 0$.

Lemma 12 (see [24]). For an $N \times N$ irreducible matrix $C$ with nonnegative off-diagonal elements satisfying the coupling condition $\sum_{j=1}^{N} c_{i j}=0$, we can obtain same propositions as follows:

(1) let $\lambda$ be an Eigen value of $C$; if $\lambda \neq 0$, then $\operatorname{Re}(\lambda)<0$;

(2) $C$ has an Eigen value 0 with multiplicity 1 and the right eigenvector $\left[\begin{array}{llll}1 & 1 & \cdots & 1\end{array}\right]^{T}$;

(3) suppose that $\varphi=\left[\varphi_{1}, \varphi_{2}, \ldots, \varphi_{N}\right]^{T} \in \mathfrak{R}^{N}$ (without loss of generality; assume $\max _{i}\left\{\varphi_{i}\right\}=1$ ) is the left eigenvector of $C$ corresponding to Eigen value 0 , and $\varphi_{i}>0$, for all $i=1,2, \ldots, N$.

Lemma 13 (Gershgorin's Circle Theorem, see [24]). For an $n \times n$ matrix $C$, define $R_{i}=\sum_{j=1, i \neq j}^{n}\left|c_{i j}\right|$. And each Eigen value of $C$ is in at least one of the disks $Z:\left|Z-c_{i j}\right| \leq R_{i}$.

Lemma 14 (see [14]). For any vectors $x, y \in \mathfrak{R}^{n}$, and $\varepsilon>0$, inequality $2 x^{T} y \leq \varepsilon x^{T} x+\varepsilon^{-1} y^{T} y$ holds.

Lemma 15 (see [9]). If matrix $C=\left(c_{i j}\right)_{i, j=1}^{m}$ is an irreducible matrix with Rank $(C)=m-1$ and satisfies $c_{i j}=c_{j i} \geq 0$, if $i \neq j$, and $\sum_{j=1}^{m} c_{i j}=0$, for $i=1,2, \ldots, m$, then, all Eigen values of matrix $\bar{C}=\left(\begin{array}{ccccc}c_{11}-c & c_{12} & \cdots & c_{1 m} \\ c_{21} & c_{22} & \cdots & c_{2 m} \\ \vdots & \vdots & \ddots & \vdots \\ c_{m 1} & c_{m 2} & \cdots & c_{m m}\end{array}\right)$ are negative.

\section{Main Results}

In this section, the pinning controllers are designed for complex network according to the structure of the network topologies (resp., symmetric irreducible, asymmetric irreducible, and $m$-reducible).

Case 1 ( $C$ is a symmetric irreducible coupling matrix). If the coupling matrix $C$ is a symmetric irreducible matrix, it means that the corresponding coupling network is undirected and strongly connected. The pinning controllers are designed as follows:

$$
u_{i}(t)= \begin{cases}-c A x_{i}(t-\tau), & i=1 \\ 0, & \text { otherwise }\end{cases}
$$

where $c$ is a positive constant; that is, $c>0$.

From (5), we can know that only the first node of the complex network (1) is selected to be controlled to stabilize the complex network (1) with symmetric irreducible coupling matrix in mean square. By using controller (5), the complex 
network (1) is controlled through pinning. Then the pinned network (1) can be rewritten as follows:

$$
\begin{aligned}
d x_{i}(t)= & {\left[f\left(x_{i}(t)\right)+\sum_{j=1}^{N} \bar{c}_{i j} A x_{j}(t-\tau)\right] d t } \\
& +g\left(t, x_{i}(t), x_{i}(t-\tau)\right) d \omega(t) \quad i=1,2, \ldots N
\end{aligned}
$$

where the elements $\bar{c}_{i j}$ of matrix $\bar{C}$ are defined as follows:

$$
\bar{c}_{i j}= \begin{cases}c_{i j}-c, & i=j=1 \\ c_{i j}, & \text { otherwise. }\end{cases}
$$

Then the following theorem gives the stabilization criteria network (1).

Theorem 16. Suppose that Assumptions 2-5 hold. If there exist positive scalars $\varepsilon_{1}>0, V_{1}>0$, and $V_{2}>0$, a small positive constant $\eta$ and a diagonal positive-definite matrix $P=$ $\operatorname{diag}\left\{p_{1}, p_{2}, \ldots p_{n}\right\}>0$ such that the following linear matrix inequalities hold:

$$
\begin{gathered}
\Psi=\left[\begin{array}{cc}
\Psi_{1} & 0 \\
0 & \Psi_{2}
\end{array}\right]<0, \\
P A \geq V_{1} I_{n}, \\
P \leq V_{2} I_{n},
\end{gathered}
$$

where $\Psi_{1}=\eta P+\varepsilon_{1}^{-1} P P+\varepsilon_{1} Q Q+V_{1} \lambda_{\max }(\bar{C})+V_{2} M_{1}^{T} M_{1}$ and $\Psi_{2}=V_{1} \lambda_{\max }(\bar{C})+V_{2} M_{2}^{T} M_{2}$; then the pinning controlled network (1) with symmetric irreducible coupling matrix $C$ is globally exponentially asymptotically stable in mean square.

Proof. Consider using the following Lyapunov functional:

$$
V(t)=\sum_{i=1}^{N} e^{\eta t} x_{i}^{T}(t) P x_{i}(t),
$$

where $P=\operatorname{diag}\left\{p_{1}, p_{2}, \ldots, p_{n}\right\}$ and $\eta$ is a sufficient small positive constant. Employing Itô's formula [25], the derivative of $V(t)$ can be expressed as follows:

$$
\begin{aligned}
d V(t)= & e^{\eta t}\left\{\sum_{i=1}^{N}\left[\eta x_{i}^{T}(t) P x_{i}(t)+2 x_{i}^{T}(t) P f\left(x_{i}(t)\right)\right]\right\} d t \\
& +e^{\eta t} \sum_{i=1}^{N} 2 x_{i}^{T}(t) P \sum_{j=1}^{N} \bar{c}_{i j} A x_{j}(t-\tau) d t \\
& +e^{\eta t} \sum_{i=1}^{N} \operatorname{trace}\left[\begin{array}{c}
g^{T}\left(t, x_{i}(t), x_{i}(t-\tau)\right) P \\
\times g\left(t, x_{i}(t), x_{i}(t-\tau)\right)
\end{array}\right] d t \\
& +e^{\eta t} \sum_{i=1}^{N} 2 x_{i}^{T}(t) P g\left(t, x_{i}(t), x_{i}(t-\tau)\right) d \omega(t) .
\end{aligned}
$$

From Lemma 14 and Assumption 2, the following inequality holds:

$$
\begin{aligned}
2 x_{i}^{T} & (t) P f\left(x_{i}(t)\right) \\
& \leq \varepsilon_{1}^{-1} x_{i}^{T}(t) P P x_{i}(t)+\varepsilon_{1} f^{T}\left(x_{i}(t)\right) f\left(x_{i}(t)\right) \\
& \leq \varepsilon_{1}^{-1} x_{i}^{T}(t) P^{2} x_{i}(t)+\varepsilon_{1} x_{i}^{T}(t) Q^{T} Q x_{i}(t) \\
& =\varepsilon_{1}^{-1} x_{i}^{T}(t) P P x_{i}(t)+\varepsilon_{1} x_{i}^{T}(t) Q Q x_{i}(t) .
\end{aligned}
$$

Noticing that $P$ and $A$ are both diagonal positive-definite matrices and LMI (9), one can obtain

$$
\begin{aligned}
& \sum_{i=1}^{N} 2 x_{i}^{T}(t) P\left[\sum_{j=1}^{N} \bar{c}_{i j} A x_{j}(t-\tau)\right] \\
& \quad=\sum_{k=1}^{n} 2 p_{k} \gamma_{k}\left(\sum_{i=1}^{N} \sum_{j=1}^{N} \bar{c}_{i j} x_{i k}(t) x_{j k}(t-\tau)\right) \\
& =\sum_{k=1}^{n} 2 p_{k} \gamma_{k}\left(x^{k}(t)\right)^{T} \bar{C} x^{k}(t-\tau) \\
& \leq 2 V_{1} \lambda_{\max }(\bar{C}) \sum_{k=1}^{n}\left(x^{k}(t)\right)^{T} x^{k}(t-\tau) \\
& =2 V_{1} \lambda_{\max }(\bar{C}) \sum_{i=1}^{N} x_{i}^{T}(t) x_{i}(t-\tau) \\
& \leq V_{1} \lambda_{\max }(\bar{C}) \sum_{i=1}^{N}\left[x_{i}^{T}(t) x_{i}(t)+x_{i}^{T}(t-\tau) x_{i}(t-\tau)\right]
\end{aligned}
$$

where $x^{k}(t)=\left(x_{1 k}(t), x_{2 k}(t), \ldots, x_{N k}(t)\right)^{T}$ for $k=1,2, \ldots, n$.

From Assumption 3 and LMI (9), the following inequality can be obtained:

$$
\begin{aligned}
\operatorname{trace} & {\left[g^{T}\left(t, x_{i}(t), x_{i}(t-\tau)\right) P g\left(t, x_{i}(t), x_{i}(t-\tau)\right)\right] } \\
\leq & V_{2}\left(\left\|M_{1} x_{i}(t)\right\|^{2}+\left\|M_{2} x_{i}(t-\tau)\right\|^{2}\right) .
\end{aligned}
$$

Equality (12) can be simplified to the following due to (13)-(15):

$d V(t)$

$$
\begin{aligned}
\leq & e^{\eta t}\left\{\sum_{i=1}^{N} x_{i}^{T}(t) \Psi_{1} x_{i}(t)+\sum_{i=1}^{N} x_{i}^{T}(t-\tau) \Psi_{2} x_{i}(t-\tau)\right\} d t \\
& +e^{\eta t} \sum_{i=1}^{N} 2 x_{i}^{T}(t) \operatorname{Pg}\left(t, x_{i}(t), x_{i}(t-\tau)\right) d \omega(t) \\
= & e^{\eta t}\left\{\left[x^{T}(t) x^{T}(t-\tau)\right] \Psi x\left[\begin{array}{c}
x(t) \\
x(t-\tau)
\end{array}\right]\right\} d t \\
& +e^{\eta t} \sum_{i=1}^{N} 2 x_{i}^{T}(t) \operatorname{Pg}\left(t, x_{i}(t), x_{i}(t-\tau)\right) d \omega(t) .
\end{aligned}
$$


From (9), one can obtain the structure of the matrix $\bar{C}$; one has that $\left|\bar{c}_{11}\right|>\sum_{j=2}^{N}\left|\bar{c}_{1 j}\right|$ and $\left|\bar{c}_{i i}\right|=\sum_{j=1, j \neq i}^{N}\left|\bar{c}_{i j}\right|$ for $i=$ $2,3, \ldots, N$. By referring to Lemma 11 , we can obtain $|\bar{C}| \neq 0$. Then we can prove that $\lambda_{\max }(\bar{C})<0$ by using Lemmas 11 and 13. From Lemma 15, it is known that all Eigen values of the matrix $\bar{C}$ are negative. Then $\lambda_{\max }(\bar{C})<0$ can be obtained. Since $\lambda_{\max }(\bar{C})<0$, LMI (8) is possible.

Taking the mathematical expectation of both sides of (16), one can obtain the following:

$$
\frac{d E\{V(t)\}}{d t} \leq e^{\eta t} E\left\{\left[\begin{array}{ll}
x^{T}(t) & x^{T}(t-\tau)
\end{array}\right] \Psi\left[\begin{array}{c}
x(t) \\
x(t-\tau)
\end{array}\right]\right\} \leq 0,
$$

which implies that $E\{V(t)\} \leq E\{V(0)\}$.

Since $V(0)=\sum_{i=1}^{N} e_{i}^{T}(0) P e_{i}(0)$, the following holds:

$$
\begin{aligned}
& e^{\eta t} \lambda_{\min }(P) E\left\{\sum_{i=1}^{N} x_{i}^{T}(t) x_{i}(t)\right\} \\
& \quad \leq E\{V(t)\} \leq E\{V(0)\} \leq \lambda_{\max }(P) \times E\left\{\sum_{i=1}^{N} x_{i}^{T}(0) x_{i}(0)\right\} .
\end{aligned}
$$

Further, the following is obtained:

$$
E\left\{\left\|x_{i}(t)\right\|^{2}\right\} \leq M_{0} e^{-\eta t} E\left\{\sum_{i=1}^{N} x_{i}^{T}(0) x_{i}(0)\right\},
$$

where $M_{0}=\lambda_{\max }(P) / \lambda_{\min }(P)$.

By Definition 7 , it can be concluded that the pinning controlled complex network (3) with a symmetric irreducible coupling matrix $\bar{C}$ is globally exponentially stable in mean square, which implies that complex network (1) with symmetric irreducible coupling matrix $C$ has been globally exponentially stabilized to the origin point in mean square by injecting the single controller (7) to the first node of complex network (1). The proof of this Theorem 16 is completed.

Case 2 ( $C$ is an asymmetric irreducible coupling matrix). If the coupling matrix $C$ is an asymmetric irreducible matrix, it means that the corresponding coupling network is directed and connected.

From Definition 9, one can conclude that $\varphi=$ $\left(\varphi_{1}, \varphi_{2}, \ldots, \varphi_{N}\right)^{T}$ is the normalized left eigenvector of matrix $C$ with respect to Eigen value zero, and $\varphi_{\min }=\min _{i}\left\{\varphi_{i}\right\}$.

Theorem 17. Suppose that Assumptions 2-5 hold. If there exist positive scalars $\varepsilon_{1}>0, V_{1}>0$, and $V_{2}>0$, a small positive constant $\eta$ and a diagonal positive-definite matrix $P=$ $\operatorname{diag}\left\{p_{1}, p_{2}, \ldots, p_{n}\right\}>0$ such that the following linear matrix inequalities hold

$$
\begin{gathered}
\Omega=\left[\begin{array}{cc}
\Omega_{1} & 0 \\
0 & \Omega_{2}
\end{array}\right]<0, \\
P A \geq V_{1} I_{n}, \\
P \leq V_{2} I_{n},
\end{gathered}
$$

where $\Omega_{1}=\phi_{i} \eta P+\varepsilon_{1}^{-1} P P+\varepsilon_{1} Q Q+(1 / 2) V_{1} \beta(\bar{C})+V_{2} M_{1}^{T} M_{1}$ and $\Omega_{2}=(1 / 2) V_{1} \beta(\bar{C})+V_{2} M_{2}^{T} M_{2}$, then the controlled network (1) with asymmetric irreducible coupling matrix $C$ is globally exponentially stabilized at the origin in mean square under the controller (5).

Proof. Consider using the following Lyapunov functional:

$$
V(t)=\sum_{i=1}^{N} \phi_{i} e^{\eta t} x_{i}^{T}(t) P x_{i}(t),
$$

where $P=\operatorname{diag}\left\{p_{1}, p_{2}, \ldots, p_{n}\right\}$. Employing Itô's formula [26], the derivative of $V(t)$ can be expressed as follows:

$$
\begin{aligned}
d V(t)= & e^{\eta t}\left\{\sum_{i=1}^{N}\left[\begin{array}{c}
\phi_{i} \eta x_{i}^{T}(t) P x_{i}(t) \\
+2 \phi_{i} x_{i}^{T}(t) P f\left(x_{i}(t)\right)
\end{array}\right]\right\} d t \\
& +e^{\eta t} \sum_{i=1}^{N} 2 \phi_{i} x_{i}^{T}(t) P \sum_{j=1}^{N} \bar{c}_{i j} A x_{j}(t-\tau) d t \\
& +e^{\eta t} \sum_{i=1}^{N} \operatorname{trace}\left[\begin{array}{c}
g^{T}\left(t, x_{i}(t), x_{i}(t-\tau)\right) \\
\phi_{i} P g\left(t, x_{i}(t), x_{i}(t-\tau)\right)
\end{array}\right] d t \\
& +e^{\eta t} \sum_{i=1}^{N} 2 \phi_{i} x_{i}^{T}(t) \operatorname{Pg}\left(t, x_{i}(t), x_{i}(t-\tau)\right) d \omega(t) .
\end{aligned}
$$

From Lemma 14 and Assumption 2, one can obtain

$$
\begin{aligned}
2 \phi_{i} x_{i}^{T}(t) P f\left(x_{i}(t)\right) \\
\quad \leq \varepsilon_{1}^{-1} x_{i}^{T}(t) P P x_{i}(t)+\varepsilon_{1} f^{T}\left(x_{i}(t)\right) f\left(x_{i}(t)\right) \\
\leq \varepsilon_{1}^{-1} x_{i}^{T}(t) P^{2} x_{i}(t)+\varepsilon_{1} x_{i}^{T}(t) Q^{T} Q x_{i}(t) \\
\quad=\varepsilon_{1}^{-1} x_{i}^{T}(t) P P x_{i}(t)+\varepsilon_{1} x_{i}^{T}(t) Q Q x_{i}(t) .
\end{aligned}
$$

Noticing that $P$ and $A$ are both diagonal positive-definite matrices and LMI (21), one can obtain

$$
\begin{aligned}
& \sum_{i=1}^{N} 2 \phi_{i} x_{i}^{T}(t) P\left[\sum_{j=1}^{N} \bar{c}_{i j} A x_{j}(t-\tau)\right] \\
& \quad=\sum_{k=1}^{n} p_{k} \gamma_{k}\left(x^{k}(t)\right)^{T}\left(\Phi \bar{C}+\bar{C}^{T} \Phi\right) x^{k}(t-\tau) \\
& \quad \leq V_{1} \beta(\bar{C}) \sum_{i=1}^{N} x_{i}^{T}(t) x_{i}(t-\tau) \\
& \quad \leq \frac{1}{2} V_{1} \beta(\bar{C}) \sum_{i=1}^{N}\left[x_{i}^{T}(t) x_{i}(t)+x_{i}^{T}(t-\tau) x_{i}(t-\tau)\right],
\end{aligned}
$$

where $x^{k}(t)=\left(x_{1 k}(t), x_{2 k}(t), \ldots, x_{N k}(t)\right)^{T}$ for $k=1,2, \ldots, n$. 
From Assumption 3 and LMI (22), the following inequality can be obtained:

$$
\begin{aligned}
& \operatorname{trace}\left[g^{T}\left(t, x_{i}(t), x_{i}(t-\tau)\right) \phi_{i} P g\left(t, x_{i}(t), x_{i}(t-\tau)\right)\right] \\
& \leq V_{2}\left(\left\|M_{1} x_{i}(t)\right\|^{2}+\left\|M_{2} x_{i}(t-\tau)\right\|^{2}\right) .
\end{aligned}
$$

Due to (25)-(27), one can simplify (24) as follows:

$$
\begin{aligned}
& d V(t) \\
& \leq e^{\eta t}\left[\sum_{i=1}^{N} x_{i}^{T}(t) \Omega_{1} x_{i}(t)+\sum_{i=1}^{N} x_{i}^{T}(t-\tau) \Omega_{2} x_{i}(t-\tau)\right] d t \\
& +e^{\eta t} \sum_{i=1}^{N} 2 \phi_{i} x_{i}^{T}(t) \operatorname{Pg}\left(t, x_{i}(t), x_{i}(t-\tau)\right) d \omega(t) \\
& =e^{\eta t}\left\{\left[x^{T}(t) x^{T}(t-\tau)\right] \Omega\left[\begin{array}{c}
x(t) \\
x(t-\tau)
\end{array}\right]\right\} d t \\
& +e^{\eta t} \sum_{i=1}^{N} 2 \phi_{i} x_{i}^{T}(t) P g\left(t, x_{i}(t), x_{i}(t-\tau)\right) d \omega(t) .
\end{aligned}
$$

From (9), one can obtain the structure of the matrix $\bar{C}$; one has that $\left|\bar{c}_{11}\right|>\sum_{j=2}^{N}\left|\bar{c}_{1 j}\right|$ and $\left|\bar{c}_{i i}\right|=\sum_{j=1, j \neq i}^{N}\left|\bar{c}_{i j}\right|$ for $i=2,3, \ldots, N$. By referring to Lemma 12 , we can obtain that $\varphi_{i}>0$ for $i=1,2,3, \ldots, N$, and $\sum_{j=1}^{N} c_{j i} \varphi_{j}=0$. Let $\Phi=\operatorname{diag}\left\{\varphi_{1}, \varphi_{2}, \ldots, \varphi_{N}\right\}$, and $K=\Phi \bar{C}+\bar{C}^{T} \Phi$ with corresponding elements $k_{i j}=\varphi_{i} \bar{c}_{i j}+\bar{c}_{j i} \varphi_{j}$. Therefore, we can obtain $\sum_{j=2}^{N}\left|k_{i j}\right|<\left|k_{11}\right|$ and $\sum_{j=1, j \neq i}^{N}\left|k_{i j}\right|=\left|k_{i i}\right|$, for $i=2,3, \ldots, N$.

Since $\bar{C}$ is irreducible and $\varphi_{i}>0$ for $i=1,2,3, \ldots, N$, matrix $K$ is also irreducible. By referring to Lemma 11, we can obtain $|K| \neq 0$. Then we can prove that $\lambda_{\max }(K)<0$ by using Lemma 11 and Lemma 13. From Lemma 15, it is known that all Eigen values of the matrix $\bar{C}$ are negative. Then $\beta(\bar{C})=$ $\lambda_{\text {max }}(K)<0$ can be obtained. Since $\beta(\bar{C})<0$, LMI (22) is possible.

Taking the mathematical expectation of both sides of (30), one can obtain the following:

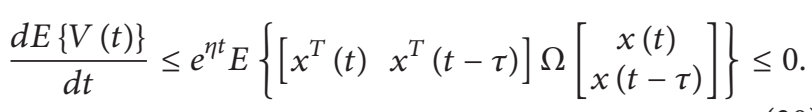

By following the proof of Theorem 16 and referring to Definition 7, it can be concluded that the controlled complex network (1) with an asymmetric irreducible coupling matrix $\bar{C}$ and with controller (5) is globally exponentially stable on the origin point in mean square, which implies that complex network (1) with asymmetric irreducible coupling matrix $C$ has been globally exponentially stabilized by using a single controller. The proof of Theorem 17 is completed.

Case 3 ( $C$ is an $m$-reducible coupling matrix). If the coupling matrix $C$ is an $m$-reducible matrix, it means that the corresponding coupling network is weakly connected. Therefore, it is not able to control complex network (1) to the origin point in mean square by using only one controller. It is important for us to design the minimum number of controllers to pinning control network (1).

From Definition 10, a complex network with $m$-reducible coupling matrix can be disconnected. For example, let $m=2$, $C_{i, k+2}=0$ for $i=1,2, \ldots, k$, and let $C_{i, k+1} \neq 0$ for $i=$ $1,2, \ldots, k$. Then we can make the corresponding network with such coupling matrix disconnect with two components. Therefore, any network can be represented by an $m$-reducible matrix, which implies that the result in this section is quite general.

By referring to [16], the irreducible matrices $C_{r}=\left(c_{i j}^{r}\right) \epsilon$ $\mathfrak{R}^{q r \times q r}, N_{0}=0$, and $N_{r}=\sum_{k=1}^{r} q k$ for $r=1,2, \ldots, k+$ $m$, the complex network error with $m$-reducible coupling matrix, can be decomposed into the following $k+m$ error subnetworks, which can be denoted by $S_{r}=\left\{N_{r-1}+1, N_{r-1}+\right.$ $\left.2, \ldots, N_{r}\right\}$ for $r=1,2, \ldots, k+m$. Similarly to equality (6), it can be noted that the set $S_{r}$ contains all the row indexes relative to the states error of $r$ th subnetwork for $r=$ $1,2, \ldots, k+m$ :

$$
\begin{aligned}
S_{r}: d x_{i}(t)= & f\left(x_{i}(t)\right) d t+g\left(t, x_{i}(t), x_{i}(t-\tau)\right) d \omega(t) \\
& +\sum_{j \in S_{r}} c_{i j} A x_{j}(t-\tau) d t \\
& +\sum_{j \in S_{v}, v>r} c_{i j} A x_{j}(t-\tau) d t, \\
& i \in S_{r}, r=1,2, \ldots, k, \\
S_{\omega}: d x_{i}(t)= & f\left(x_{i}(t)\right) d t+g\left(t, x_{i}(t), x_{i}(t-\tau)\right) d \omega(t) \\
& +\sum_{j \in S_{\omega}} c_{i j} A x_{j}(t-\tau) d t \\
& i \in S_{\omega}, \omega=k+1, k+2, \ldots, k+m .
\end{aligned}
$$

From [16], we can conclude that the error subnetwork for $k+1 \leq \omega \leq k+m$ is independent from the rest of the error subnetworks. And the error sub-network $S_{r}$ for $1 \leq$ $r \leq k$ is influenced by the error subnetwork $S_{s}$ for $s>r$. Therefore not less than $m$ controllers is needed for the pinning synchronization of the $m$ self-governed error subnetworks $S_{\omega}$ for $k+1 \leq \omega \leq k+m$, which implies that at least $m$ controllers are required to stabilize complex network (1) with $m$-reducible coupling matrix.

$M$ controllers are designed to exponentially stabilize complex network (1) with $m$-reducible coupling matrix. The first node of each error subnetwork $S_{\omega}$ for $k+1 \leq \omega \leq k+m$ is chosen to be controlled, and the $m$ controllers are designed as follows:

$$
u_{i}(t)= \begin{cases}-c A x_{i}(t-\tau), & i=N_{\omega-1}+1, \\ & \omega=k+1, \ldots, k+m \\ 0, & \text { otherwise. }\end{cases}
$$


Then, we can obtain the error networks by using the controllers (33) to control complex network (1), which can be described by (31) and the following:

$$
S_{\omega}:\left\{\begin{aligned}
& d x_{N_{\omega-1}+1}(t)= f\left(x_{i}(t)\right) d t \\
&+g\left(t, x_{i}(t), e_{i}(t-\tau)\right) d \omega(t) \\
&+\sum_{j \in S_{\omega}} c_{N_{\omega-1}+1, j} A x_{j}(t-\tau) \\
&-c A x_{N_{\omega-1}+1}(t-\tau) d t \\
& d x_{i}(t)= f\left(x_{i}(t)\right) d t \\
&+g\left(t, x_{i}(t), e_{i}(t-\tau)\right) d \omega(t) \\
&+\sum_{j \in S_{\omega}} c_{i j} A x_{j}(t-\tau) d t \\
& i \neq N_{\omega-1}+1, i \in S_{\omega}, \\
& \omega=k+1, k+2, \ldots, k+m .
\end{aligned}\right.
$$

Let matrix $\bar{C}_{r}=C_{r}$ for $1 \leq r \leq k$ and for $k+1 \leq \omega \leq k+m$ matrix $\bar{C}_{\omega}=\left(\bar{c}_{i j}^{\omega}\right)_{q \omega \times q \omega}$ such that the elements $\bar{c}_{i j}^{\omega}$ are defined as follows:

$$
\bar{c}_{i j}= \begin{cases}c_{i j}-c, & i=j=N_{\omega-1}+1 \\ c_{i j}, & \text { otherwise }\end{cases}
$$

where $i, j \in S_{\omega}$.

Then, the error subnetworks (36) can be rewritten as

$$
\begin{aligned}
S_{\omega}: d x_{i}(t)= & f\left(x_{i}(t)\right) d t+g\left(t, x_{i}(t), x_{i}(t-\tau)\right) d \omega(t) \\
& +\sum_{j \in S_{\omega}} \bar{c}_{i j} A x_{j}(t-\tau) d t \\
& \quad i \in S_{\omega}, \omega=k+1, k+2, \ldots, k+m .
\end{aligned}
$$

Let matrices $\widetilde{C}_{S}=\left(\widetilde{c}_{i j}^{s}\right)_{q s \times q s}$ be such that

$$
\begin{aligned}
& \tilde{c}_{i j}^{s}=c_{i j}^{s} \quad i \neq j, \\
& \tilde{c}_{i i}^{s}=-\sum_{j=1, j \neq i}^{N} c_{i j}^{s} \quad i=1,2, \ldots, q s,
\end{aligned}
$$

for $s=1,2, \ldots, k+m \cdot \varphi^{i}=\left(\varphi_{1}^{i}, \varphi_{2}^{i}, \ldots, \varphi_{q i}^{i}\right)^{T}$ denotes the normalized left eigenvector of matrix $\widetilde{C}_{i}$ with respect to Eigen value zero, and $\varphi_{\min }^{i}=\min \left\{\varphi_{1}^{i}, \varphi_{2}^{i}, \ldots, \varphi_{q i}^{i}\right\}$ for $i=1,2, \ldots, k+$ $m$.

Every subnetwork can be seen as a network with symmetric irreducible coupling matrix $C_{i}$ or asymmetric irreducible coupling matrix $C_{i}$; therefore, we can use the same way of Case 1 or Case 2. Similarly to Theorem 16 and Theorem 17, we can obtain the following.

Theorem 18. Suppose that Assumptions 2-5 hold. If there exist positive scalars $\varepsilon_{1}^{i}>0, V_{1}^{i}>0, V_{2}^{i}>0$, and a small positive constants $\eta_{i}$ a diagonal positive-definite matrix $P^{i}=$ $\operatorname{diag}\left\{p_{1}^{i}, p_{2}^{i}, \ldots, p_{n}^{i}\right\}>0$ such that the following LMIs hold

$$
\begin{gathered}
\Xi^{i}=\left[\begin{array}{cc}
\Xi_{1}^{i} & 0 \\
0 & \Xi_{2}^{i}
\end{array}\right]<0, \\
P^{i} A \geq V_{1}^{i} I_{n}, \\
P^{i} \leq V_{2}^{i} I_{n}
\end{gathered}
$$

where $\Xi_{1}^{i}=\phi_{i} \eta_{i} P^{i}+\left(\varepsilon_{1}^{i}\right)^{-1} P^{i} P^{i}+\varepsilon_{1}^{i} Q Q+(1 / 2) V_{1}^{i} \beta\left(\bar{C}_{i}\right) I_{n}+$ $V_{2}^{i} M_{1}^{T} M_{1}$ and $\Xi_{2}^{i}=(1 / 2) V_{1}^{i} \beta\left(\bar{C}_{i}\right) I_{n}+V_{2}^{i} M_{2}^{T} M_{2}$, and $\beta\left(\bar{C}_{i}\right)$ is given in Definition 9, then the controlled network (1) with an $m$-reducible coupling matrix $C$ is globally exponentially at the drive network (1) in mean square.

Proof. For $k+1 \leq i \leq k+m$, since LMIs (38) hold, from Theorem 17, it can be concluded that every controlled subnetwork with symmetric irreducible coupling matrix $C_{i}$ or asymmetric irreducible coupling matrix $C_{i}$ is globally exponentially at the drive subnetwork in mean square; that is, the error subnetworks $S_{\omega}(k+1 \leq \omega \leq k+m)$ can be globally exponentially synchronized. It means that the states of network (1) can be constringed to 0 ; that is, $\lim _{t \rightarrow \infty} x(t) \rightarrow$ 0 .

Therefore complex network (1) with $m$-reducible coupling matrix can be stabilized to the origin point by designing at least $m$ controllers. The proof of Theorem 18 is completed.

\section{Numerical Examples}

In this section, two examples are given to show the effectiveness of the proposed synchronization scheme. The networks are composed of ten coupled nodes, and each node satisfies the chaotic Lorenz system as its dynamics. A chaotic Lorenz system can be described as follows:

$$
\dot{x}_{i}=f\left(x_{i}\right)=\left(\begin{array}{ccc}
-a & a & 0 \\
b & -1 & 0 \\
0 & 0 & -c
\end{array}\right)\left(\begin{array}{l}
x_{i 1} \\
x_{i 2} \\
x_{i 3}
\end{array}\right)+\left(\begin{array}{c}
0 \\
-x_{i 1} x_{i 3} \\
x_{i 1} x_{i 2}
\end{array}\right),
$$

where $a, b$, and $c$ are parameters. Let $a=10, b=28$, and $c=8 / 3$; the system can have a chaotic attractor. The attractor of the first node is shown in Figure 1. Figure 1(a) shows the attractor of the three states of the first node without the noise perturbation, and Figure 1(b) shows the attractor with the same initial condition and with the noise perturbation. And referring to [13], we can obtain that Assumption 2 is satisfied by using this chaotic Lorenz system.

Example 1. We consider a complex network consisting of ten nodes which are described by Lorenz system. Assume that 


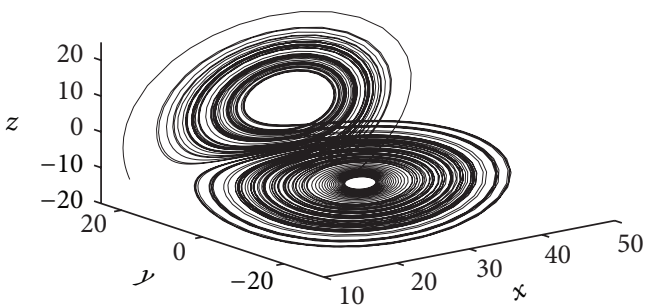

(a)

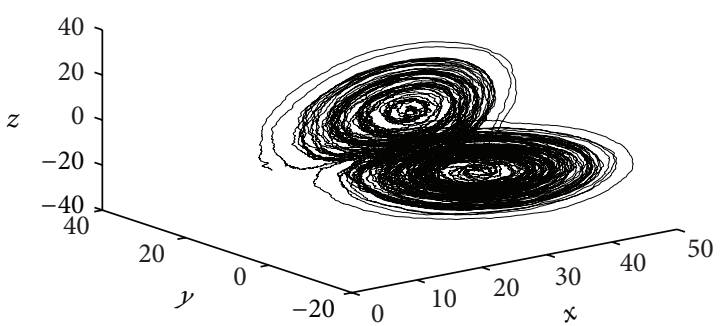

(b)

FIGURE 1: The attractor of the three states of the first nodes.

the network is coupled with symmetric and irreducible matrix $C$ satisfying Case 1 . Matrix $C$ is described as follows:

$$
C=\left(\begin{array}{cccccccccc}
-3 & 1 & 0 & 0 & 0 & 0 & 0 & 1 & 0 & 1 \\
1 & -2 & 1 & 0 & 0 & 0 & 0 & 0 & 0 & 0 \\
0 & 1 & -2 & 1 & 0 & 0 & 0 & 0 & 0 & 0 \\
0 & 0 & 1 & -2 & 1 & 0 & 0 & 0 & 0 & 0 \\
0 & 0 & 0 & 1 & -3 & 1 & 0 & 0 & 0 & 1 \\
0 & 0 & 0 & 0 & 1 & -2 & 1 & 0 & 0 & 0 \\
0 & 0 & 0 & 0 & 0 & 1 & -2 & 1 & 0 & 0 \\
1 & 0 & 0 & 0 & 0 & 0 & 1 & -3 & 1 & 0 \\
0 & 0 & 0 & 0 & 0 & 0 & 0 & 1 & -2 & 1 \\
1 & 0 & 0 & 0 & 1 & 0 & 0 & 0 & 1 & -3
\end{array}\right)
$$

The inner connection matrix is taken as an identity matrix of dimension 3, that is, $A=I_{3}$.

In simulation, we assume that the coupling delay of the network is $\tau=50$ and the noise intensity function is $\sigma\left(t, x_{i}(t), X(t-\tau)\right)=0.1 x_{i}(t)+0.02 \sum_{k=1}^{10} x_{k}(t-\tau)$. And the initial states of the $i$ th node of the networks are

$$
\begin{aligned}
& \left(x_{i 1}(t), x_{i 2}(t), x_{i 3}(t)\right) \\
& \quad=(10+0.1 i,-6+0.1 i, 3+0.1 i), \quad t \in[-\tau, 0] .
\end{aligned}
$$

The controlling gain of the only one controller is taken as $c=0.02$, and the time delay of the controller is $\tau=$ 50. Figure 2 shows the trajectories of the states $x_{i k}(t)(k=$ $1,2,3 ; i=1,2, \ldots, 10)$ of the complex networks. It can be concluded that all of the states $x_{i k}(t)(k=1,2,3 ; i=$ $1,2, \ldots, 10)$ tend to zero, which implies that a complex network coupled with symmetric and irreducible matrix is pinning controlled to the origin point by utilizing only one controller.

Example 2. We assume that the network is composed of ten Lorenz nodes coupling with asymmetric and irreducible matrix $C$ satisfying Case 2. Matrix $C$ is as follows:

$$
C=\left(\begin{array}{cccccccccc}
-3 & 1 & 0 & 0 & 0 & 0 & 0 & 1 & 0 & 1 \\
1 & -2 & 1 & 0 & 0 & 0 & 0 & 0 & 0 & 0 \\
1 & 0 & -2 & 1 & 0 & 0 & 0 & 0 & 0 & 0 \\
0 & 0 & 1 & -2 & 1 & 0 & 0 & 0 & 0 & 0 \\
0 & 0 & 0 & 1 & -3 & 1 & 0 & 0 & 0 & 1 \\
0 & 0 & 0 & 0 & 1 & -2 & 1 & 0 & 0 & 0 \\
0 & 0 & 0 & 0 & 0 & 1 & -2 & 1 & 0 & 0 \\
1 & 0 & 0 & 0 & 0 & 0 & 1 & -3 & 1 & 0 \\
0 & 0 & 0 & 0 & 0 & 0 & 0 & 1 & -2 & 1 \\
0 & 0 & 1 & 0 & 1 & 0 & 0 & 0 & 1 & -3
\end{array}\right)
$$

The other conditions are consistent with Example 1. The controlling gain of the only one controller is taken as $c=0.02$, and time delay of the controller is $\tau=50$. Figure 3 shows the trajectories of the states $x_{i k}(t)(k=1,2,3 ; i=1,2, \ldots, 10)$ of the complex networks. It can be concluded that all of the states $x_{i k}(t)(k=1,2,3 ; i=1,2, \ldots, 10)$ tend to zero, which implies that a complex network coupled with asymmetric and irreducible matrix is pinning controlled to the origin point by utilizing only one controllers.

Example 3. A complex network is coupled with matrix $C$ satisfying Case 3; that is, $C$ is an $m$-reducible matrix. Matrix $C$ is described as follows:

$$
C=\left(\begin{array}{cccccccccc}
-7 & 3 & 0 & 4 & 0 & 0 & 0 & 0 & 0 & 0 \\
2 & -2 & 0 & 0 & 0 & 0 & 0 & 0 & 0 & 0 \\
0 & 0 & -5 & 3 & 2 & 0 & 0 & 0 & 0 & 0 \\
0 & 0 & 2 & -6 & 4 & 0 & 0 & 0 & 0 & 0 \\
0 & 0 & 3 & 1 & -4 & 0 & 0 & 0 & 0 & 0 \\
0 & 0 & 0 & 0 & 0 & -5 & 5 & 0 & 0 & 0 \\
0 & 0 & 0 & 0 & 0 & 3 & -3 & 0 & 0 & 0 \\
0 & 0 & 0 & 0 & 0 & 0 & 0 & -3 & 1 & 2 \\
0 & 0 & 0 & 0 & 0 & 0 & 0 & 1 & -2 & 1 \\
0 & 0 & 0 & 0 & 0 & 0 & 0 & 2 & 2 & -4
\end{array}\right)
$$

In simulation, the other conditions are consistent with Example 1. Since $C$ is an $m$-reducible coupling matrix, we can obtain $m=3$ by referring to Definition 10; therefore we need at least three controllers to pinning control the complex network. And we select the controllers (33) to control the 
Journal of Control Science and Engineering

9
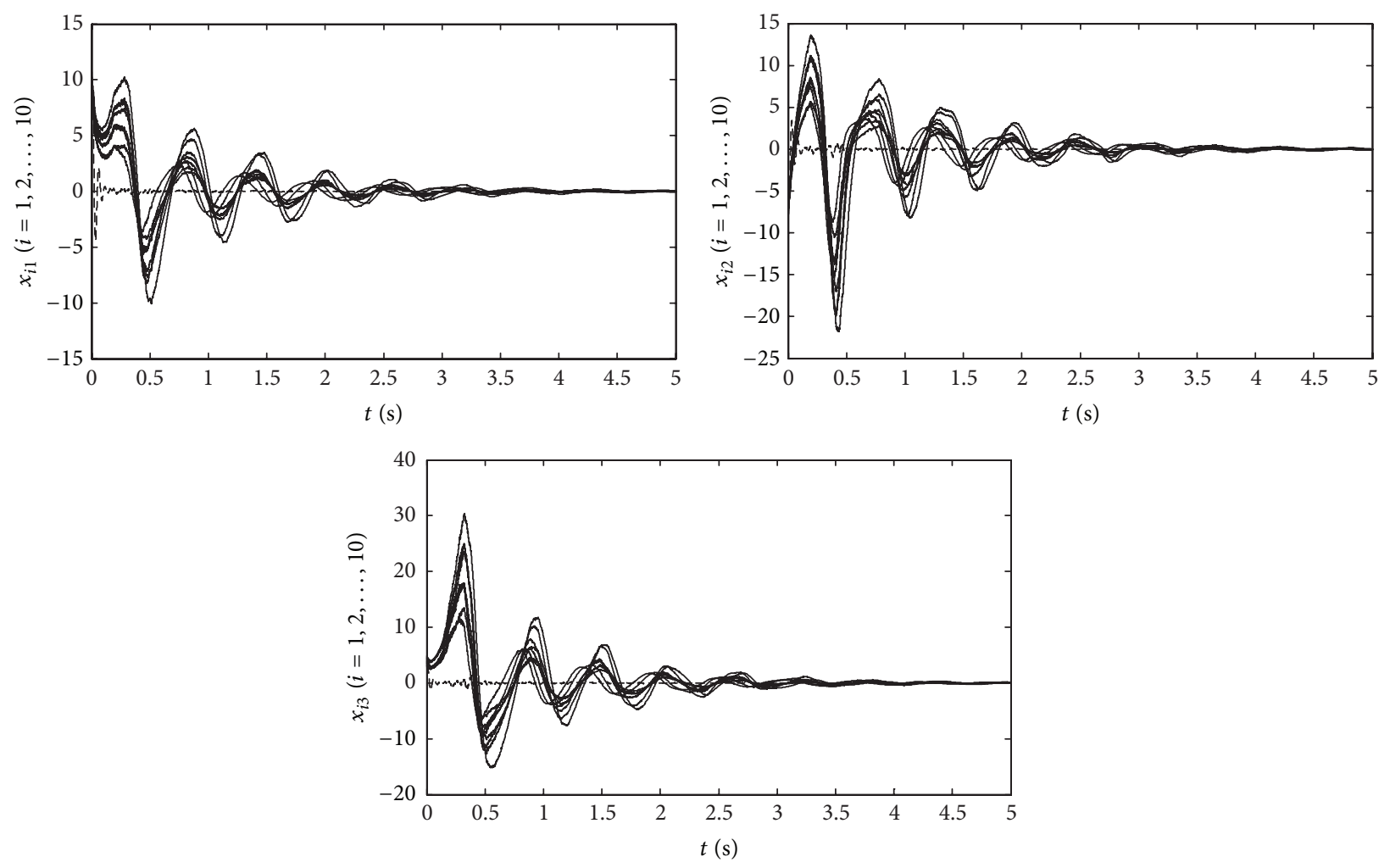

FIgURE 2: The states of complex network with a symmetric irreducible coupling matrix $C$.
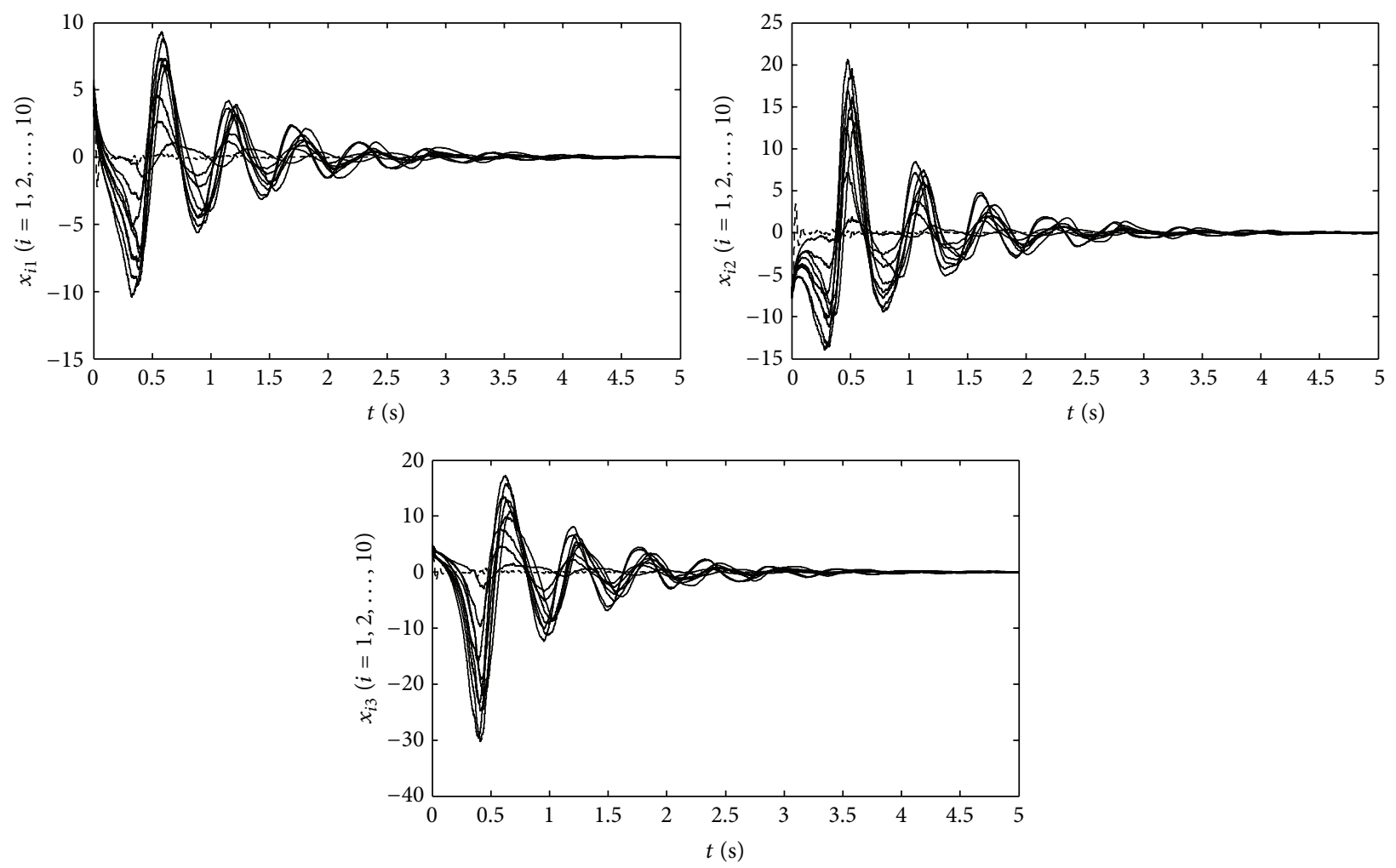

FIGURE 3: The states of complex network coupled with an asymmetic and irreducible matrix. 

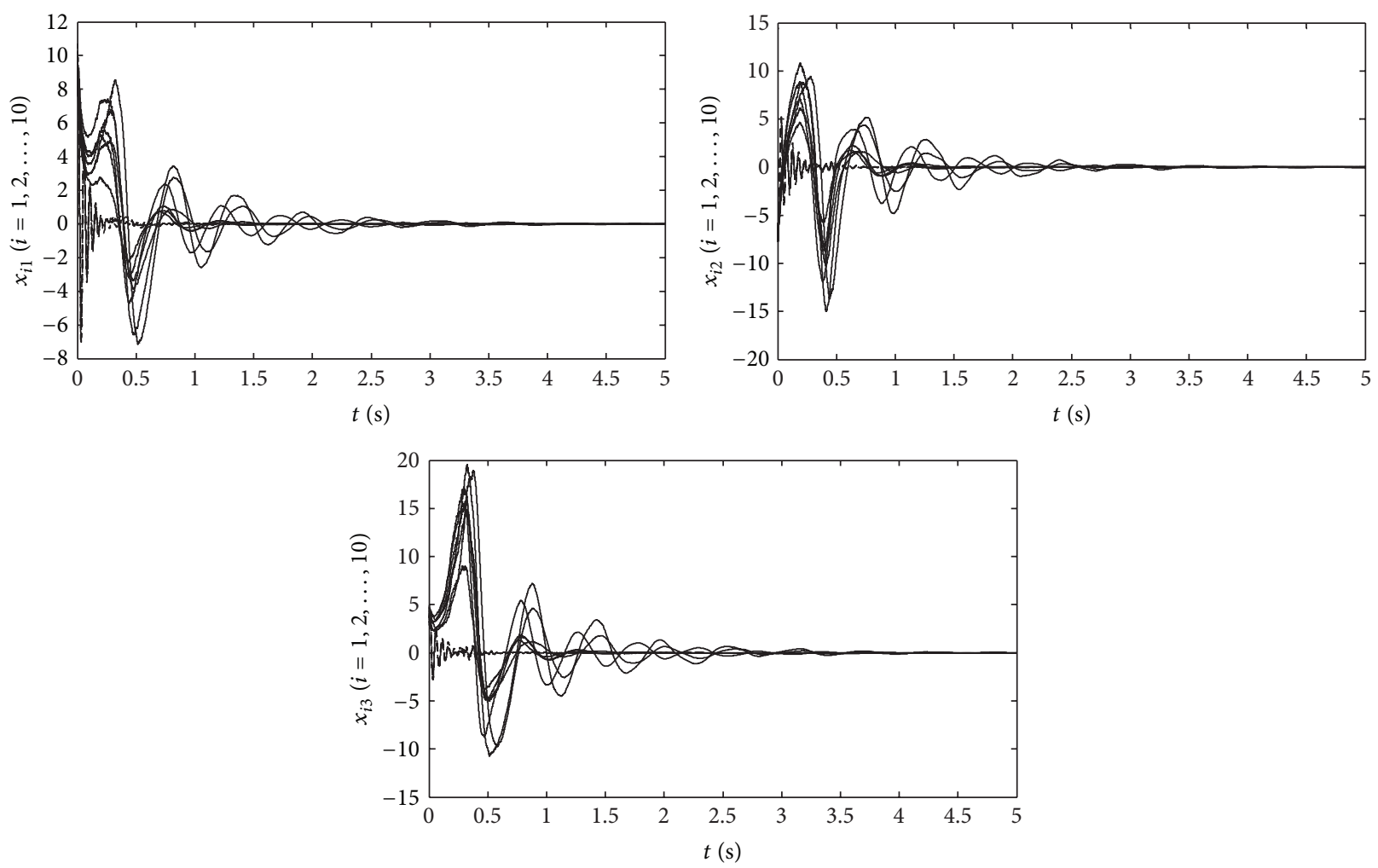

FIgURE 4: The states of complex networks with an $m$-reducible coupling matrix $C$.

complex network, which further means that the nodes 3, 6 , and 8 (first node of subnetworks 2, 3, and 4) are to be controlled. And the controlling gains of the three controllers are all taken as $c=0.026$, and the time delays of the controllers are also taken as $\tau=50$. Figure 4 shows ten trajectories of the stabilized states $x_{i k}(t)(k=1,2,3 ; i=$ $1,2, \ldots, 10)$ of the complex network. It can be concluded that all of the states $x_{i k}(t)(k=1,2,3 ; i=1,2, \ldots, 10)$ tend to zero, which implies that the complex networks coupled with reducible matrix can be stabilized by utilizing the minimize number of controllers.

Remark 19. It can be inferred that the stabilization criteria obtained in Theorems 16-18 are effective for complex networks coupled with time delays and disturbed by the stochastic noise. Only one controller is used for a connected network to stabilize it. For an unconnected network, we can firstly portion it as several connected subnetworks; then only one controller is used for the connected subnetwork and therefore the unconnected network is stabilized by the minimal number of controllers.

\section{Conclusion}

The stabilization problem of time-delayed coupling complex networks with a stochastic perturbation by utilizing pinning controllers is studied in this paper. A minimal number of controllers are designed to force the states of complex networks to the origin point in mean square by fully using the structure of the network topology matrix. Some stabilization criteria are achieved to pinning control the complex network, which are described in the form of LMIs. Some examples are given to show the effectiveness of the proposed pinning controller in this paper.

\section{Conflict of Interests}

The authors declare that there is no conflict of interests regarding the publication of this paper.

\section{Acknowledgments}

This work was supported in part by the National Natural Science Foundation of China under 61104103, 61203028, and 61374180, the Natural Science Foundation for Colleges and Universities in Jiangsu Province, China, under 10KJB120001, and Climbing Program of Nanjing University of Telecommunications \& Posts, China, under NY210013 and NY210014.

\section{References}

[1] X. Li and G. Chen, "Synchronization and desynchronization of complex dynamical networks: an engineering viewpoint," IEEE Transactions on Circuits and Systems I: Fundamental Theory and Applications, vol. 50, no. 11, pp. 1381-1390, 2003.

[2] X. F. Wang, "Complex networks: topology, dynamics and synchronization," International Journal of Bifurcation and Chaos 
in Applied Sciences and Engineering, vol. 12, no. 5, pp. 885-916, 2002.

[3] I. Belykh, V. Belykh, and M. Hasler, "Synchronization in complex networks with blinking interactions," in Proceedings of the International Conference on Physics and Control (PhysCon '05), pp. 86-91, August 2005.

[4] G.-P. Jiang, W. K.-S. Tang, and G. Chen, "A state-observer-based approach for synchronization in complex dynamical networks," IEEE Transactions on Circuits and Systems I: Regular Papers, vol. 53, no. 12, pp. 2739-2745, 2006.

[5] M. Chavez, D.-U. Hwang, and S. Boccaletti, "Synchronization processes in complex networks," European Physical Journal: Special Topics, vol. 146, no. 1, pp. 129-144, 2007.

[6] G. Solís-Perales, E. Ruiz-Velázquez, and D. Valle-Rodríguez, "Synchronization in complex networks with distinct chaotic nodes," Communications in Nonlinear Science and Numerical Simulation, vol. 14, no. 6, pp. 2528-2535, 2009.

[7] W. Yu, G. Chen, and J. Lü, "On pinning synchronization of complex dynamical networks," Automatica, vol. 45, no. 2, pp. 429-435, 2009.

[8] X. F. Wang and G. Chen, "Pinning control of scale-free dynamical networks," Physica A: Statistical Mechanics and its Applications, vol. 310, no. 3-4, pp. 521-531, 2002.

[9] T. Chen, X. Liu, and W. Lu, "Pinning complex networks by a single controller," IEEE Transactions on Circuits and Systems I: Regular Papers, vol. 54, no. 6, pp. 1317-1326, 2007.

[10] Z. X. Liu, Z. Q. Chen, and Z. Z. Yuan, "Pinning control of weighted general complex dynamical networks with time delay," Physica A: Statistical Mechanics and its Applications, vol. 375, no. 1, pp. 345-354, 2007.

[11] L. Xiang, Z. Chen, Z. Liu, F. Chen, and Z. Yuan, "Pinning control of complex dynamical networks with heterogeneous delays," Computers and Mathematics with Applications, vol. 56, no. 5, pp. 1423-1433, 2008.

[12] Y. Wu, W. Wei, G. Li, and J. Xiang, "Pinning control of uncertain complex networks to a homogeneous orbit," IEEE Transactions on Circuits and Systems II: Express Briefs, vol. 56, no. 3, pp. 235239, 2009.

[13] X. Li, X. Wang, and G. Chen, "Pinning a complex dynamical network to its equilibrium," IEEE Transactions on Circuits and Systems I: Regular Papers, vol. 51, no. 10, pp. 2074-2087, 2004.

[14] J. Lu, D. W. C. Ho, and Z. Wang, "Pinning stabilization of linearly coupled stochastic neural networks via minimum number of controllers," IEEE Transactions on Neural Networks, vol. 20, no. 10, pp. 1617-1629, 2009.

[15] J. Zhou, J.-A. Lu, and J. Lü, "Pinning adaptive synchronization of a general complex dynamical network," Automatica, vol. 44, no. 4, pp. 996-1003, 2008.

[16] C. P. Li, W. G. Sun, and J. Kurths, "Synchronization of complex dynamical networks with time delays," Physica A: Statistical Mechanics and its Applications, vol. 361, no. 1, pp. 24-34, 2006.

[17] H. R. Karimi and H. Gao, "New delay-dependent exponential Ho synchronization for uncertain neural networks with mixed time delays," IEEE Transactions on Systems, Man, and Cybernetics B: Cybernetics, vol. 40, no. 1, pp. 173-185, 2010.

[18] H. Zhang, Y. Shi, and J. Wang, "Observer-based tracking controller design for networked predictive control systems with uncertain Markov delays," International Journal of Control, vol. 86, no. 10, pp. 1824-1836, 2013.

[19] Z. Shuai, H. Zhang, J. Wang, J. Li, and M. Ouyang, "Combined AFS and DYC control of four-wheel-independent-drive electric vehicles over can network with time-varying delays," IEEE Transactions on Vehicular Technology, vol. 63, no. 2, pp. 591-602, 2014.

[20] J. Cao, P. Li, and W. Wang, "Global synchronization in arrays of delayed neural networks with constant and delayed coupling," Physics Letters A: General, Atomic and Solid State Physics, vol. 353, no. 4, pp. 318-325, 2006.

[21] L. Arnold, Stochastic Differential Equations: Theory and Applications, Wiley-Interscience, New York, NY, USA, 1974.

[22] Z. Wang, Y. Liu, M. Li, and X. Liu, "Stability analysis for stochastic Cohen-Grossberg neural networks with mixed time delays," IEEE Transactions on Neural Networks, vol. 17, no. 3, pp. 814-820, 2006.

[23] H. Huang, D. W. C. Ho, and J. Lam, "Stochastic stability analysis of fuzzy Hopfield neural networks with time-varying delays," IEEE Transactions on Circuits and Systems II: Express Briefs, vol. 52, no. 5, pp. 251-255, 2005.

[24] R. A. Horn and C. R. Johnson, Matrix Analysis, Cambridge University Press, Cambridge, UK, 1990.

[25] O. Taussky, "A recurring theorem on determinants," The American Mathematical Monthly, vol. 56, no. 10, pp. 672-676, 1949.

[26] X. Mao, Stochastic Differential Equations and Their Applications, Horwood, Chichester, UK, 1997. 

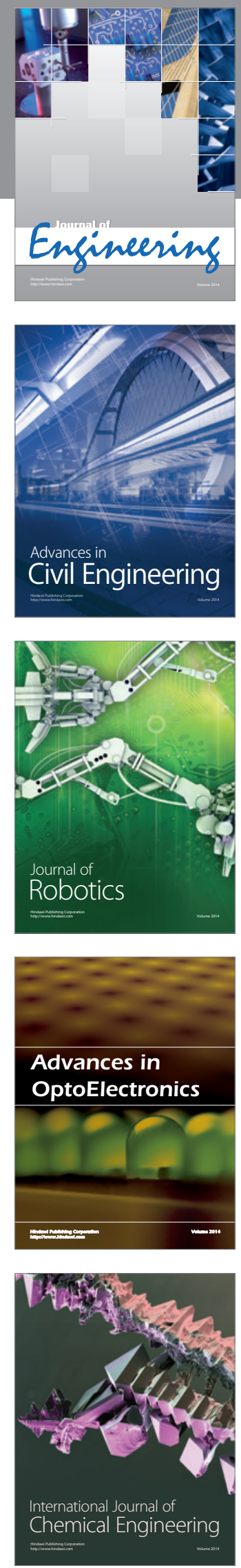



The Scientific World Journal
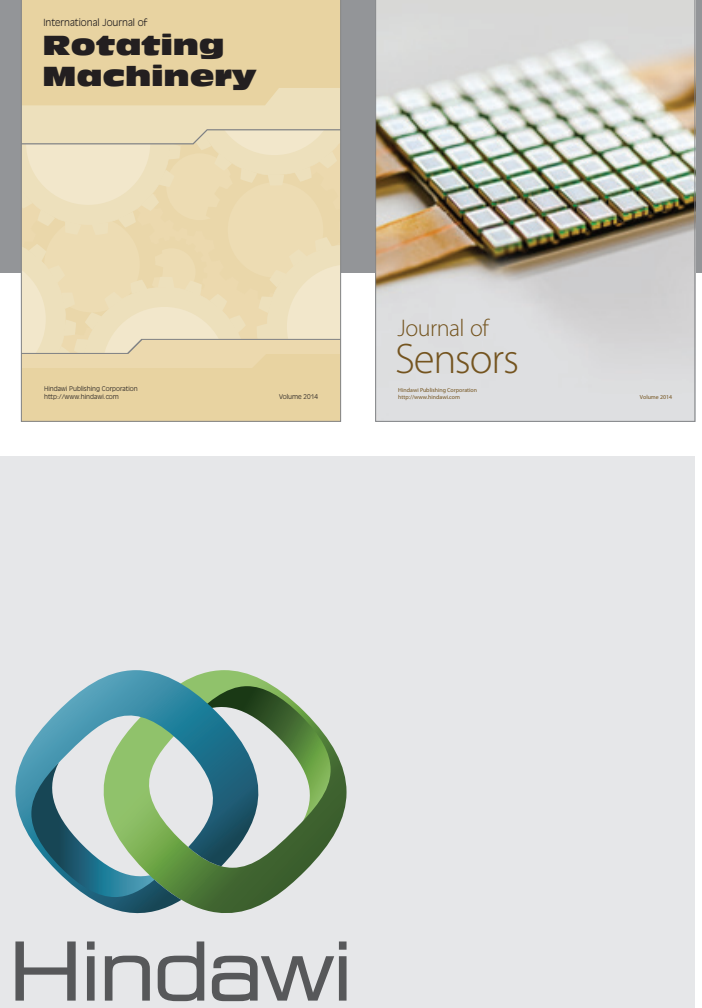

Submit your manuscripts at http://www.hindawi.com
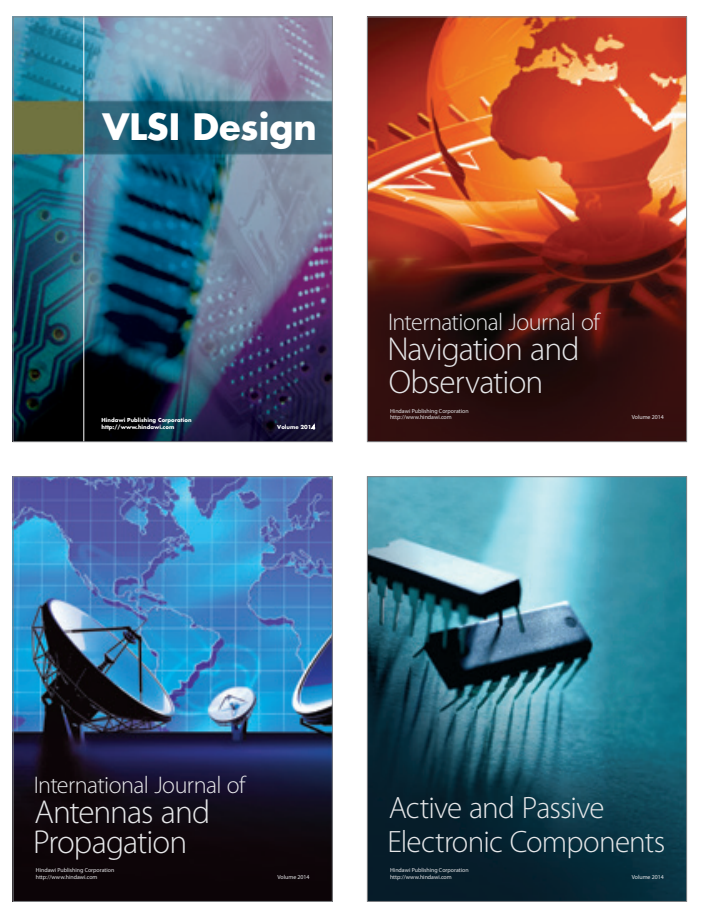


Journal of

Control Science

and Engineering
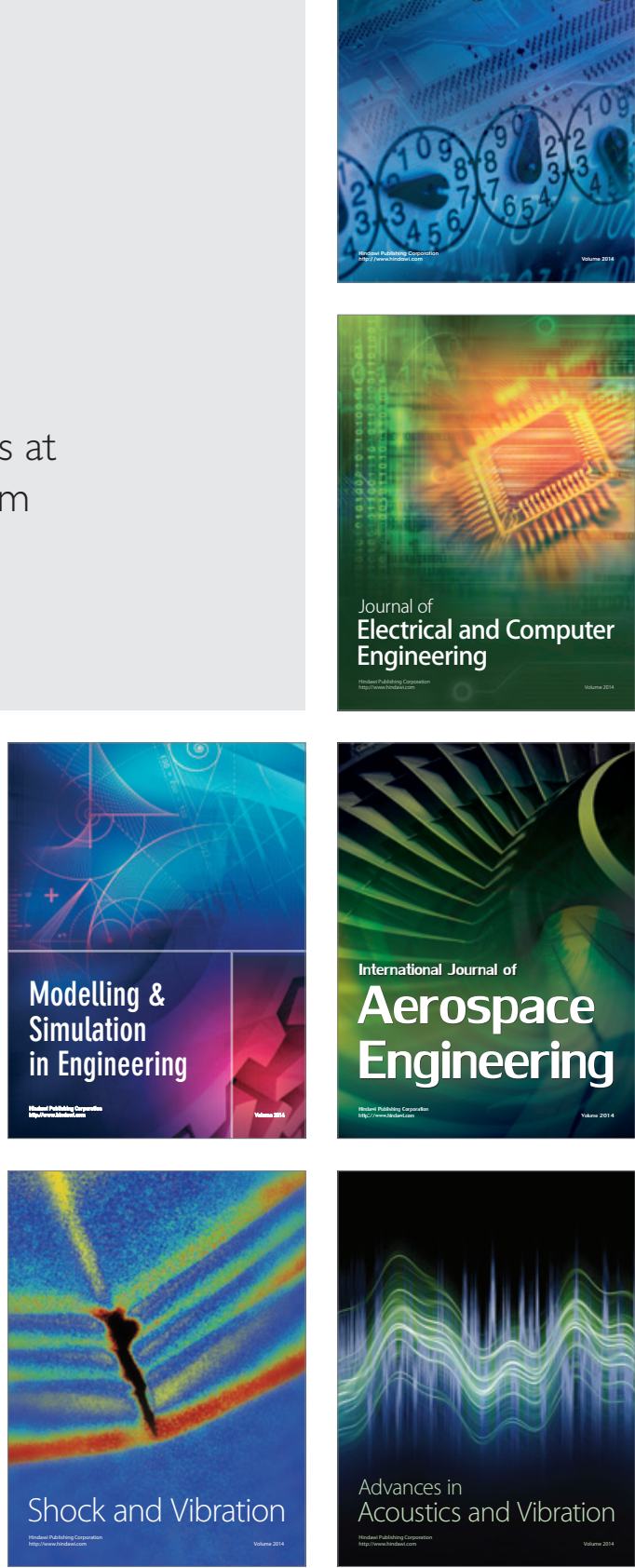